\title{
Comparing Bender-Gestalt Test and Quantitative Electroencephalography for Brain Trauma Diagnosis in Depressive and Attention Deficit Hyperactivity Disorders
}

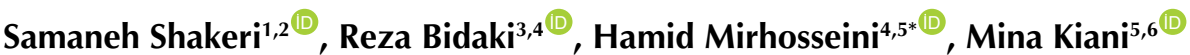 \\ 'Department of Psychology, Islamic Azad University of Yazd, Yazd, Iran \\ ${ }^{2}$ PHD Student in Family and sexual Health, Shahed University, Tehran, Iran \\ ${ }^{3}$ Diabetes Research Center, Shahid Sadoughi University of Medical Sciences, Yazd, Iran \\ ${ }^{4}$ Research Center of Addiction and Behavioral Sciences, Shahid Sadoughi University of Medical \\ Sciences, Yazd, Iran \\ ${ }^{5}$ Student Research Committee, Shahid Sadoughi University of Medical Sciences, Yazd, Iran \\ ${ }^{6}$ Mehravaran Rehabilitation Center for Chronic Mental Patients, State Welfare Organization, Abadeh, \\ Fars, Iran
}

\begin{abstract}
Background: Some experts assert there is an association between traumatic brain injury and cognitive impairments such as attention deficit hyperactivity disorder (ADHD) and depressive disorder. Furthermore, children and adults with ADHD struggle with focusing, organizing tasks, and feeling restless. They might experience sadness, guilt, irritability, low self-confidence and helplessness. In this regard, ADHD and depressive disorder occasionally occur together. We aimed to compare the clinical application of the Bender-Gestalt test (BGT) and electroencephalography in screening brain damage in the patients with the mentioned disorders.

Methods: This was a cross-sectional study to assess diagnostic accuracy. Eighty patients with depression $(n=35)$ and ADHD $(n=45)$ resulting from brain damage aged 10-35 years who had been referred by the psychologist or psychiatrist to Imam Hossein Clinic in Yazd. Both the BGT and quantitative electroencephalography (QEEG) results existed in their files. Patients with any serious medical, other psychiatric disorders or history of drug dependency were excluded. Paired $t$ test was used to analyze the differences.

Results: The brain damage score was different in the two tests. Based on the paired $t$ test, the BGT was a more valid screening test. Also, the score of the two tests were different in the depression and ADHD mean scores $(P<0.05)$.

Conclusion: BGT was a more reliable tool compared to QEEG for diagnosing brain damage within the patients with the mentioned disorders.

Keywords: Bender-Gestalt test; Quantitative electroencephalography; Brain damage; Depressive disorder; Attention deficit hyper activity disorder.
\end{abstract}

*Correspondence to Hamid Mirhosseini. Address: Department of Psychiatry, Taft, Yazd Tel: +98(353)2632006S Email: Mirhosseini@ssu.ac.ir

Published online June 30 2021

Citation: Shakeri S, Bidaki R, Mirhosseini H, Kiani M. Comparing bender-gestalt test and quantitative electroencephalography for brain trauma diagnosis in depressive and attention deficit hyperactivity disorders. Clin Neurosci J. 2021;8(3):144-148. doi:10.34172/ icnj.2021.29.

\section{Introduction}

In accidents, the head is exposed to the most damage and it is the most common cause for hospitalization and mortality (40\%-50\%) in injured people in different incidents. ${ }^{1}$ Currently, the longtime consequences of brain damage are recognized more than before. These problems are mostly physical, cognitive, emotional and behavioral which might be aggravated significantly after brain damage. ${ }^{2}$ Clinical experience confirms this fact that some of the neuropsychiatric symptoms can also be seen in organic brain damages. ${ }^{3}$ Overlaps between the mentioned subjects have led the psychological clinics to use special tests called neuropsychology tests for differential diagnosis. ${ }^{4}$ The Bender-Gestalt test (BGT) is one of the simplest types of neuropsychological tests. ${ }^{5}$ It is considered as a current assessment tool for visualmotor integration assessment. ${ }^{2}$ Considering the growing interest of neuropsychiatrists in the applicability of this tool for diagnosing organic causes and on the other hand, incompatibility of research results, it seems that more studies need to be conducted and their results should be carefully analyzed. ${ }^{6,7}$

(C) 2021 The Author(s). This is an open access article distributed under the terms of the Creative Commons Attribution License (http:// creativecommons.org/licenses/by/4.0/), which permits unrestricted use, distribution, and reproduction in any medium, provided the original work is properly cited. 
People who experience head damage are exposed to the risk of depression and cognitive disorders; but patients, their families and also the therapeutic teams seem to be more concerned about the physical problems, whereas depression and attention deficit hyperactivity disorders (ADHD) are significantly growing. Depression is the most common mood disorder and the most important psychiatric disorder of the present century. ${ }^{8}$ Psychiatric disorders such as depressive disorders are not accompanied by a tangible damage to the human brain and nervous system; There has always been this assumption that psychiatric disorders are only the consequences of psychological processes and they are not related to nervous phenomena. However, following the advances in functional neuroimaging, the abnormal activity of neural circuits in the brain is responsible for many psychiatric disorders. ${ }^{9}$ Another disorder is ADHD which has caught the attention of psychiatrists as a common disorder in childhood with obvious symptoms of impulsivity. Follow-up studies imply the continuity of the disorder up to adolescence and adulthood and psychological disturbances in other life stages. Amongst etiology hypotheses regarding this disorder and based on conducted studies, a delay in growth or abnormal morphology of the frontal cortex has been mentioned. Neural histology studies emphasize that the companionship of neuropsychiatric cognitive battery tests with other clinical studies such as functional and structural neuroimaging can give us a more comprehensive view and understanding of this disorder. Head injury can create a set of neuropsychiatric symptoms and even lead to dementia or psychiatric disorders. ${ }^{10}$ These patients are prone to progression and exacerbation of depressive symptoms and a suitable evaluation and treatment modality should be considered. Moreover, after returning to the society, they might suffer from different disorders such as Alzheimer's or dementia and other illnesses that can impair interpersonal relationships and lead to losing job opportunities and performance capacity or possible isolation and withdrawal. ${ }^{11}$

A study on the effectiveness of teaching neurofeedback on brain waves pattern of children suffering from ADHD, showed that neurofeedback treatment affects the brain wave pattern of these children. ${ }^{12}$ Another study on the effectiveness of teaching stress coping skills on parenting stress of mothers whose child suffered from ADHD, showed that an intervention modality could reduce the parenting stress of these mothers. ${ }^{13}$

Grover and colleagues studied the acute effects of near infrared light therapy on brain state in healthy subjects as quantified by quantitative electroencephalography (QEEG) measures. They concluded that there was no significant difference between the groups under treatment. ${ }^{14}$ In another study on defining abnormal slow EEG activity in acute ischemic stroke, delta/alpha ratio as an optimal QEEG index, the researchers concluded that these two were significant indices..$^{15}$ One study showed that the EEG pattern in patients with brain lesion was significantly different from healthy subjects. ${ }^{16}$

So far and based on our reviews, the difference between the BGT and QEEG in brain damage has not been analyzed. Also, the possibility of the existence of other alternative tests for distinguishing differences in brain damage is one of the reasons for this research. In this regard, this study can play a significant role in the field of neuropsychiatry about brain damage, BGT, and QEEG. BGT and QEEG can help psychologists, neurologists, neuropsychiatrists, and other researchers determine the role of these two modalities in enhancing the physical and mental health of the patients after a traumatic brain damage. Also, the results of this study persuade therapists to use these tests because of their availability and being inexpensive. Considering the importance of early and primitive diagnosis of patients with brain injury, a measuring tool or device for early diagnosis of brain lesions seems essential in order to help the experts. Thus, we aimed to assess the difference between the BGT and QEEG in brain lesion diagnosis.

\section{Materials and Methods}

This was an analytical-cross sectional study comparing the diagnostic accuracy of the BGT and QEEG in screening patients with brain damage.

\section{Statistical Population; Sample and Sampling}

Among the patients with ADHD and depression following mild brain injury aged 10-35 years with Glasgow-Coma score more than 13 referred to Imam Hossein Clinic in Yazd, 80 people ( 35 with depression and 45 with ADHD) were selected. The sample size was similar to a similar study. ${ }^{17}$ The order of using the tests (BGT, QEEG) in patients was completely random.

\section{Inclusion Criteria}

- Age of 10-35 years

- Diagnosed with depression and ADHD by a psychologist

- Absence of any important physical neurological disease

\section{Exclusion Criteria}

- Addiction (drug/substance dependency)

- Considerable neurological diseases

- Other major psychiatric disorders

\section{Research Tools}

$B G T$ : Visual-motor BGT, usually known as the BGT or only Bender test, is used as a screening tool for studying probable brain trauma. Clinical experience shows that some psychiatric disorders overlap with symptoms 
related organic brain lesions, leading to the use of neuropsychological tests for differential diagnosis. The BGT is one of the most famous neuropsychological tests which is easy to use. ${ }^{3}$ It is one of the most successful tests which neuropsychologists and neurologists used to evaluate brain damages. ${ }^{18}$ It can be used easily and quickly in revealing a person's perspective and approach toward his or her world.

Despite successful experiences, it faces some limitations and precautions. BGT is only able to relatively detect the extent of damage mostly in the right hemisphere and it is unable to detect tiny brain damages in the left hemisphere. ${ }^{19}$ Another problem is that there is no uniform scoring and interpretation system approved by all experts. ${ }^{5}$

The test was given to the examinees and we asked them to draw shapes in white papers measuring $11 \times 8.5$ inches. The drawing was assessed in terms of integrity and accuracy. A study on 474 healthy people (control group) and 187 people with neurosis and 136 people with psychosis declared that reliability coefficient of the BGT was 0.7 with the retest method. ${ }^{7}$

QEGG: Electroencephalography could be used for research purposes to diagnose brain injury. EEG is the digital or paper recording of brain waves. The human brain is an electrochemical system. Brain electrical activities can be transmitted on the skull surface. These activities are very weak and on a microvolt scale. EEG machine records them by electrodes connected to the skull and shows it in brain waves. ${ }^{20-22}$ QEEG provides quantitative information on brain functions. ${ }^{23}$ It creates live and dynamic maps of the brain and compares them to normal data. QEEG provides additional information to many clinical diagnoses. ${ }^{24}$ It is a valuable diagnostic application for cerebrovascular diseases of the brain, brain damages, ADHD, learning disorders, anxiety, depression, brain tumors, epilepsy, schizophrenia, and Alzheimer's disease. ${ }^{25}$ It has 0.97 sensitivity and 0.84 specificities for distinction between several psychiatric disorders. ${ }^{26}$

\section{Data Analysis Method}

- Descriptive Statistical Methods (such as average, standard deviation, minimum and maximum score)

- Paired $t$ test to analyze the differences between options
- $\quad$ SPSS software, version 21 , was used to analyze the data. The significance level of 0.05 was considered for all the hypotheses.

\section{Results}

\section{Descriptive Findings}

In order to inform the dispersion of the subjects' scores in each of the variables, the scores, average index and standard deviations were shown separately. Also, 35 people had depression and 45 had ADHD. According to Kolmogorov-Smirnov test and $P<>0.05$, there was no significant difference between two groups and they have a normal distribution (Table 1).

\section{Inferential Findings}

Cohen' kappa coefficient was applied to determine the correlation between brain damage score and BGT/QEEG values. There was a significant and positive relation according to 0.99 confidence level. $(P<0.01, r=0.67)$ There was a significant and positive relation between BGT and QEEG values with depression according to $99 \%$ confidence $(P<0.01, \mathrm{r}=0.65)$. Also, in the correlation test, there was a significant and positive relation between ADHD scores with BGT and QEEG values according to $99 \%$ confidence $(P<0.01, \mathrm{r}=0.67)$.

\section{Analyzing Paired t Assumptions}

There was no significant relation between two groups and they were statistically comparable. The mean scores for the diagnosis of brain injury by BGT and QEEG were 0.362 and 0.200 based on paired $t$ test and $P=0.0001$ (Table 2).

The mean BGT and QEEG scores for the depressed people were 0.38 and 0.2 , respectively $(P=0.012$, Table 2 ). The mean QEEG and BGT scores for patients with ADHD were 0.2 and 0.35 , respectively $(P=0.007$, Table 2$)$.

\section{Discussion}

This study showed that the BGT is more accurate than QEEG for screening brain damage in ADHD and depressive disorders. This is consistent with the findings of Mirzavand et al, ${ }^{7}$ Sheikhi, ${ }^{3}$ Saravani, ${ }^{6}$ Grover et al, ${ }^{14}$ and Thornton \& Carmody ${ }^{16}$ in which BGT was successfully was used for assessing 81 to 120 patients with brain trauma compared with healthy individuals. QEEG

Table 1. Descriptive Data of the Research Variables

\begin{tabular}{|c|c|c|c|c|}
\hline Variables & Sample Size & Minimum Age & Maximum Age & Mean (SD) \\
\hline Bender-Gestalt test scores for depression & 35 & 10 & 35 & $0.38(0.40)$ \\
\hline QEEG scores for depression & 35 & 10 & 35 & $0.21(0.41)$ \\
\hline Bender-Gestalt test scores for hyperactivity & 45 & 10 & 35 & $0.36(0.41)$ \\
\hline QEEG scores for hyperactivity & 45 & 10 & 35 & $0.20(0.40)$ \\
\hline
\end{tabular}

Abbreviation: SD, standard deviation.

The subjects' scores in each of the variables, the scores of average index and standard deviation, are shown separately. 
Table 2. Comparing Mean Bender-Gestalt Test Scores with QEEG Scores in the Two Groups

\begin{tabular}{lcccc}
\hline Variable & Test & Mean $(\mathrm{SD})$ & $\mathbf{t}$ & $\boldsymbol{P}$ Value \\
\hline \multirow{2}{*}{ Brain damage } & BGT & $0.36(0.483)$ & 3.951 & 0.0001 \\
& QEEG & $0.200(0.402)$ & & \\
\multirow{2}{*}{ Depression } & BGT & $0.38(0.49)$ & & \\
& QEEG & $0.2(0.41)$ & & 0.012 \\
ADHD & BGT & $0.355(0.484)$ & & \\
& QEEG & $0.2(0.404)$ & & 0.007 \\
\hline
\end{tabular}

Abbreviations: ADHD, attention deficit hyperactivity disorder; SD, standard deviation; BGT, Bender-Gestalt test; QEEG, quantitative electroencephalography.

Values are given as mean and (SD) for three variables to compare BGT with QEEG.

Paired $t$ test, $P<0.05$.

deficiency may be related to involuntary captured diverse artifacts especially at low frequencies which are related to brain trauma. Nevertheless, the BGT is flexible and can be interpreted by different methods.

Consistent with our results, a study regarding the clinical application of BGT compared with MRI images showed that BGT can be used for diagnosing brain traumatic injury screening in neurology clinics and to prevent some unnecessary imaging. ${ }^{27}$ Because of few disadvantages such as lack of patient compliance and technical issues, QEEG is less competent.

Application of the visual-motor BGT is more reliable compared with QEEG in the screening of brain trauma in patient with depression, while some researchers believe that QEEG is a promising screening measure among individuals with mild traumatic brain injury. ${ }^{28}$ Since the latter study was conducted on healthy and older subjects, this inconsistency may be related to patient characteristics. Moreover, QEEG findings are related to only the cerebral cortex and the information from deep brain regions are not included.

There was a significant difference between the average scores of BGT and QEEG in ADHD diagnosis; and this difference is in favor of BGT. Consistently, it has been shown that BGT was a reliable and powerful tool for detection of many cognitive impairments in the ADHD population. ${ }^{18}$

With respect to QEEG failure, it can be said that fear and anxiety in younger patients lead to unwanted artifacts that might interfere with the results. Furthermore, researchers have suggested that different brain areas have abnormalities. For example, the reticular activating system which is involved in attention and consciousness does not work properly. ${ }^{29}$ Neuroimaging analysis has shown that children with ADHD have serious problems in the cerebellum and the frontal lobe which is the region that is involved in planning, organizing, decision-making, time perception, active memory, inhibition, maintenance, and thinking. ${ }^{30}$ Regardless of what was mentioned, analysis of the body's metabolism also demonstrated that these people have distinctive differences in brain neurochemistry with other children. ${ }^{31}$

This is while the involved subcortical regions in ADHD have been ignored during electroencephalography. Besides, the relation between brain neurochemistry activity and QEEG is obscure. Considering the disadvantages of QEEG such as artifacts and excluding subcortical data, BGT may be more functional for detecting brain trauma among the patients with cognitive impairment.

\section{Conflict of Interest Disclosures}

The authors declare no conflicts of interest

\section{Authors' contributions}

Study concept, design, and student mentorship: HM. Data acquisition: SS and HM. Data analysis, interpretation of the findings: SS and RB and MK. Reviewing and revising the manuscript and approving the final version for publication: All authors.

\section{Ethical Statement}

The study was approved by the Ethics Committee of the Yazd University of Medical Sciences (ethics ID: IR.SSU.MEDICINE. REC.1396.177) and followed ethical considerations of the declaration of Helsinki.

\section{Financial Support}

This study had no funding support.

\section{Acknowledgements}

This paper is an excerpt of a MSc thesis in clinical psychology from Islamic Azad University of Yazd, Iran.

\section{References}

1. Ansari M, Bayan L, Gorji A. The impact of road accidents on brain injury. Shefaye Khatam. 2016;4(3):103-10. doi: 10.18869/acadpub.shefa.4.3.103. [Persian].

2. Wan L, Thomas Z, Pisipati S, Jarvis SP, Boutros NN. Inhibitory deficits in prepulse inhibition, sensory gating, and antisaccade eye movement in schizotypy. Int J Psychophysiol. 2017;114:47-54. doi: 10.1016/j. ijpsycho.2017.02.003.

3. Sheikhi S. Clinical application if Bender-Gestalt Test in screening brain damages and comparing them with MRI results. Journal of Urmia Nursing and Midwifery Faculty. 2007;5(1):12-4. [Persian].

4. Ganji H. Psychological Tests. Tehran: Salavan Publishing; 2015. [Persian].

5. Groth-marnat G. Handbook of Psychological Assessment: For Clinical Psychologists, Therapists and Psychotherapists. Trans. by Pasha Sharifi H, Nikkhoo M. Roshd Publishing; 2005.

6. Saravani RM. Analyzing the Validity and Reliability of Bender-Gestalt Test in Brain Damage Diagnosis with Electroencephalogram [thesis]. Tehran: Allameh Tabataba’i University; 1994. [Persian]. 
7. Mirzavand J, Tarafi S, Zarinfar A, Zarinfar S. Comparing the Diagnosis Ability of Bender-Gestalt and Description Electroencephalography in Brain Lesions. National Conference on 4 Corners on Humanities; 2015; Shiraz.

8. Kleinke $\mathrm{CH}$. Coping with life challenges: Anxiety, depression, loneliness, shyness, failure, marital problem, deprivation, old age, pain, sickness, damage, loss. Trans. by Mohammadkhani SH. Special Media Publishing. 2016.

9. Montazeri A, Mousavi SJ, Omidvari S, Tavousi M, Hashemi A, Rostami T. Depression in Iran: a systematic review of the literature (2000-2010). Payesh. 2013;12(6):567-94. [Persian].

10. Sadock B, Sadock V, Ruiz P. Summary of Kaplan and Sadock's Psychology. Trans. by Ganji M. Arjmand Publishing; 2003.

11. Seel RT, Kreutzer JS, Rosenthal M, Hammond FM, Corrigan JD, Black K. Depression after traumatic brain injury: a National Institute on Disability and Rehabilitation Research Model Systems multicenter investigation. Arch Phys Med Rehabil. 2003;84(2):177-84. doi: 10.1053/ apmr.2003.50106.

12. Besharat MA, Hamidi S, Rostami R, Farahani H. The effectiveness of neurofeedback training on EEG among children with attention deficit/hyperactivity disorder. Contemporary Psychology. 2012;7(1):3-16. [Persian].

13. Behpazhouh A, Motevalipour A, Farzad V, Rostami R, Habibi Askarabadi M. The efficacy of stress coping skills training on parenting stress of mothers with children suffering from attention deficit hyperactivity disorder. J Fam Res. 2010;6(21):99-114. [Persian].

14. Grover F Jr, Weston J, Weston M. Acute effects of near infrared light therapy on brain state in healthy subjects as quantified by QEEG measures. Photomed Laser Surg. 2017;35(3):136-41. doi: 10.1089/pho.2015.4036.

15. Finnigan S, Wong A, Read S. Defining abnormal slow EEG activity in acute ischaemic stroke: delta/alpha ratio as an optimal QEEG index. Clin Neurophysiol. 2016;127(2):1452-9. doi: 10.1016/j.clinph.2015.07.014.

16. Thornton KE, Carmody DP. Traumatic brain injury rehabilitation: QEEG biofeedback treatment protocols. Appl Psychophysiol Biofeedback. 2009;34(1):59-68. doi: 10.1007/s10484-009-9075-4.

17. Delavar A. Method of Research in Psychology and Egucational Science. Tehran: Virayesh Publishing;

18. Koppitz EM. The Bender Gestalt Test for Young Children.. New York: Grune \& Stratton; 1964.

19. Ganji H, Sabet M. Psychometrics: Theoretical Basis of Psychological Tests. Savalan Publishing; 2017. [Persian].

20. Vecchiato G, Astolfi L, De Vico Fallani F, Toppi J, Aloise
F, Bez F, et al. On the use of EEG or MEG brain imaging tools in neuromarketing research. Comput Intell Neurosci. 2011;2011:643489. doi: 10.1155/2011/643489.

21. Astolfi L, De Vico Fallani F, Cincotti F, Mattia D, Bianchi L, Marciani MG, et al. Neural basis for brain responses to TV commercials: a high-resolution EEG study. IEEE Trans Neural Syst Rehabil Eng. 2008;16(6):522-31. doi: 10.1109/ tnsre.2008.2009784.

22. Ambler T, Ioannides A, Rose S. Brands on the brain: neuroimages of advertising. Bus Strateg Rev. 2000;11(3):17-30. doi: 10.1111/1467-8616.00144.

23. Chaturvedi M, Bogaarts JG, Kozak Cozac VV, Hatz F, Gschwandtner U, Meyer A, et al. Phase lag index and spectral power as QEEG features for identification of patients with mild cognitive impairment in Parkinson's disease. Clin Neurophysiol. 2019;130(10):1937-44. doi: 10.1016/j.clinph.2019.07.017.

24. Oraki M, Vise Tehrani N. The comparision of effectiveness of biofeedback and neuro biofeedback training on reduction of headache migraine. Neuropsychology. 2017;2(7):107-18. [Persian].

25. Fielnz M, Mories R. Neuroscience: Science of the Brain: An Introduction for Young Students. Trans. by Sour B, Panahi R, Shahbazi A. Ensan Publishing; 2016.

26. Chabot RJ, Merkin H, Wood LM, Davenport TL, Serfontein G. Sensitivity and specificity of QEEG in children with attention deficit or specific developmental learning disorders. Clin Electroencephalogr. 1996;27(1):26-34. doi: $10.1177 / 155005949602700105$.

27. Ghalehban M, Besharat MA, Rad E. The use of the bender visual motor gestalt test with substance abuse patients. Procedia Soc Behav Sci. 2011;30:433-6. doi: 10.1016/j. sbspro.2011.10.085.

28. Ianof JN, Anghinah R. Traumatic brain injury: an EEG point of view. Dement Neuropsychol. 2017;11(1):3-5. doi: 10.1590/1980-57642016dn11-010002.

29. Kinomura S, Larsson J, Gulyás B, Roland PE. Activation by attention of the human reticular formation and thalamic intralaminar nuclei. Science. 1996;271(5248):512-5. doi: 10.1126/science.271.5248.512.

30. Giedd JN. Structural magnetic resonance imaging of the adolescent brain. Ann N Y Acad Sci. 2004;1021:77-85. doi: 10.1196/annals.1308.009.

31. Madani A, Heydari-Nasab L, Yaghoubi H, Rostami R. The Effectiveness of Neurofeedback with Cognitive Tasks on Attention Deficit / Hyperactivity (ADHD Symptoms) in Adulthood. J Clin Psychol. 2015;7(4):59-70. doi: 10.22075/ jcp.2017.2218. [Persian] 